\title{
Schach von Wuthenow: Wuthenow oder Venedig Schachs Reisefantasie im Fontaneschen Kontext
}

Schach von Wuthenow stirbt, wie man weiß, durch Selbstmord in seinem Wagen an der Ecke Behren- und Wilhelmstraße in Berlin, und zwar so allein, daß nicht einmal der Leser an seinem Ende teilnimmt. Er bleibt bei Kutscher und Groom auf dem Bock und hört den tödlichen Schuß von dorther. Ja, er wird noch mehr von dem schrecklichen Geschehen in der Kutsche distanziert, denn beide Bedienstete kommentieren den zunächst noch mysteriösen Knall in der ihnen vertrautesten, dem Leser aber ungewohnten Sprache: der Groom auf Englisch und der Kutscher auf Plattdeutsch; in Worten übrigens, die in dem berühmten Anfang von Thomas Manns Buddenbrooks wieder aufgenommen sein könnten: " Damn<, sagte der Groom. >What's that? > Wat et is? Wat soll et sind, Kleener?«(129)

Schach also stirbt in Berlin - und doch sind, bevor er die Tat vollbringt, in seiner eigenen Fantasie mit seinem Tod ganz andere, viel exotischere Orte verbunden, von denen als Alternative zum heimischen Wuthenow zunächst vor allem einer erscheint: Venedig. Schon bei den Hochzeitsvorbereitungen im 18. Kapitel bemerkt Frau von Carayon, die Hochzeit selbst solle zwar in ihrer Wohnung stattfinden, aber

Was dann weiter zu geschehen habe, das stehe bei dem jungen Paare; sie sei neugierig, ob Venedig über Wuthenow oder Wuthenow über Venedig den Sieg davontragen werde. Die Lagunen hätten sie gemeinsam und die Gondel auch, und nur um eines müsse sie bitten, daß der kleine Brückensteg unterm Schilf, an dem die Gondel liege, nie zur Seufzerbrücke werde. (123)

Gegen Ende des Kapitels hat dann am Vorabend der Hochzeit »aller halben Widerrede der Frau von Carayon zum Trotz« Venedig endgültig »über Wuthenow gesiegt $\ll$. (124)

Anknüpfend an diese Bemerkung verliert sich nun Schach in eine Reisefantasie, die in der Sekundärliteratur bisher kaum gewürdigt worden ist. ${ }^{1} \mathrm{Da}$ bei entfaltet der Schluss des 18. Kapitels von Fontanes Roman ein reizvolles Spiel mit Realität und Imagination, bei dem der Hinweis- und Deutungscharakter des Fantasierten für die Ereignisse in der Wirklichkeit das Zentrum bildet. Fontane bedient sich einer indirekten und symbolischen Aussageweise, bei der die Bilder und Visionen, denen Schach sich hingibt, Aufschluß über seine seelische Verfassung und sein zukünftiges Schicksal geben. 
Worum handelt es sich? Schach ist im Begriff, dem Befehl seines Königs zu folgen und Victoire von Carayon zu heiraten, die er trotz ihrem von Blatternarben gezeichneten Gesicht verführt hat. Die Hochzeitsvorbereitungen werden getroffen, und den Abend vor der Trauung verbringt Schach im Hause der Braut, wo er das so naheliegende Thema im Gespräch zu meiden versucht: "Er war heitrer und in seinem Urteile milder als sonst, und vermied nur in ebenso bemerkenswerter wie zum Glück unbemerkt bleibender Weise, von der Hochzeit und den Vorbereitungen dazu zu sprechen. « Lebhaft, »um so plauderhafter« wird er erst,

wenn das Gespräch auf die Reisetage nach der Hochzeit hinüberlenkte. Denn Venedig, aller halben Widerrede der Frau von Carayon zum Trotz, hatte doch schließlich über Wuthenow gesiegt, und Schach, wenn die Rede darauf kam, hing mit einer ihm sonst völlig fremden Phantastik allen erdenklichen Reiseplänen und Reisebildern nach. Er wollte nach Sizilien hinüber und die Sireneninsel passieren, >ob frei oder an den Mast gebunden, überlaß er Victoiren und ihrem Vertrauen sie nach Malta. Nicht um Maltas willen, o nein. Aber auf dem Wege dahin sei die Stelle, wo der geheimnisvolle schwarze Weltteil in Luftbildern und Spiegelungen ein allererstes Mal zu dem in Nebel und Schnee gebornen Hyperboreer spräche. Das sei die Stelle, wo die bilderreiche Fee wohne, die stumme Sirene, die mit dem Zauber ihrer Farbe fast noch verführerischer locke, als die singende. Beständig wechselnd seien die Szenen und Gestalten ihrer Laterna magica, und während eben noch ein ermüdeter Zug über den gelben Sand ziehe, dehne sich's plötzlich wie grüne Triften, und unter der schattengebenden Palme säße die Schar der Männer, die Köpfe gebeugt und alle Pfeifen in Brand, und schwarz und braune Mädchen, ihre Flechten gelöst und wie zum Tanze geschürzt, erhüben die Becken und schlügen das Tamburin. Und mitunter sei's, als lach' es. Und dann schwieg es und schwänd es wieder. Und diese Spiegelung aus der geheimnisvollen Ferne, das sei das Ziel!

Und Victoire jubelte, hingerissen von der Lebhaftigkeit seiner Schilderung.

Aber im selben Augenblick überkam es sie bang und düster, und in ihrer Seele rief eine Stimme: Fata Morgana.

Was auf den ersten Blick wie eine farbige Urlaubsschilderung klingt und den Leser Victoires Begeisterung teilen läßt, macht auch ihn, sobald er den Text genauer betrachtet, nachdenklich. Schachs Vision schwindet am Schluß auf bestürzende Weise, und der Leser beginnt zu begreifen: Dies ist Schachs Todesfantasie, nicht nahe Zukunft, nicht Flitterwochenwirklichkeit, sondern Fata Morgana, wie die hellsichtige Braut spürt, denn längst ist Schach ent- 
schlossen, sich der fatalen Ehe durch den Freitod zu entziehen. Seit wann? Seit er sich im 14. Kapitel auf seinen Familiensitz Wuthenow zurückgezogen hat. Das letzte Wort dieses von Vaget analysierten Kapitels ${ }^{2}$ ist, »Nein, nein«, Schachs innere Antwort auf die Ehebilder mit Victoire, die er sich ausmalt. Die Beziehung zwischen Schachs späterem tatsächlichen Entschluß und dieser halb unbewußten Einstimmung wird denn auch folgerichtig im 17. Kapitel hergestellt: »Ein Gedanke, den er schon in Wuthenow gefaßt hatte, kam ihm jetzt wieder und reifte rasch zum Entschluß« (120); und zu recht, wenn auch ahnungslos stellt Frau von Carayon in ihrer schon zitierten Bemerkung die Beziehung zwischen Wuthenow und Venedig her.

Eine der auffälligsten Szenen in dem Wuthenow-Kapitel ist Schachs Kahnfahrt, bei der er über den »bloße[n] tote[n] Arm des Sees « »nah an [der] Schloßterrasse « (97) hinausmuß, bevor er sich in der Strömung des eigentlichen Sees treiben lassen kann. Schon hier hat Schach den festen Boden unter den Füßen verloren. Es kostet ihn große Mühe, "an die verpaßte Stelle zurückzukommen« (98). Er hat das Wasser des Todes - wie Aschenbach in seiner Gondel auf dem Weg zum Lido - schon überquert.

Allerdings ist die Symbolik in diesem Kapitel nicht, wie Vagets Interpretation nahelegt, in sich abgeschlossen; sie entfaltet ihre Dimensionen erst in Beziehung zu Schachs Reisefantasie und zum 16. Kapitel, in dem Frau von Carayon Schritte unternimmt, Schach zur Heirat ihrer Tochter zu zwingen. Dabei muß man über Demetz' Bemerkung noch hinausgehen: »das Zurückweichen Schachs auf seinen ländlichen Zufluchtsort [...] und der leidenschaftliche Entschluß Frau von Carayons, ihn zur Legalisierung zu zwingen [...] sind einander zugeordnet; Schachs $>$ nein<, ihr >ja< schaffen die gesteigerte Situation, welche die Charaktere gleichsam im Sprung weitertreibt. ${ }^{3}$ Die wechselseitigen Bezüge dieser Kapitel sind bis ins Detail durchgeführt: Während Schach sich der Ehe zu entziehen versucht, bemüht sich Frau von Carayon, sie zu sichern. Während er sich in Wuthenow entscheidungslos im Kreise dreht, durcheilt sie auf der Suche nach dem König lange, gerade Strecken (»eine dunkle melancholische Zypressenallee schien gar kein Ende nehmen zu wollen [...] großen Mittelgang [...] immer weiter in den Park hineinzufahren«). Während er mit seinen Familienporträts Zwiesprache hält, wird sie mit Kurtisane (das Andenken an die Gräfin Lichtenau im Marmorpalais von Potsdam) und mit gefallenem Mädchen (das Bild der büßenden Magdalena in Schloß Paretz) konfrontiert. Während er an einem Orte bleibt, wechseln ihre Eindrücke ständig (Potsdam, Hotel Einsiedler, Neuer Garten usw.). Während er von der vertrauten Stätte seiner Kindheit auf das unbekannte Wasser des Todes vordringt, begegnet umgekehrt sie lauter unbekannten Eindrücken, bevor sie in dem Generaladjutanten von Köcknitz auf einen alten Bekannten trifft. Während er sich aufs Wasser begibt, das zerstörerische Wirkung auf ihn hat, fährt sie an ihm entlang (»einen neben einem See einherlaufenden Weg«), 
überquert es (»hatte man die >Wublitz , einen von Mummeln überblühten Havelarm, zu passieren) und erlebt seine beruhigende Kraft (»vergaß über diese Schönheit all ihr Leid«, 111 f.).

Schachs Reisefantasie im 18. Kapitel ist die folgerichtige Weiterentwicklung seines Verfallenseins an das Wasser, denn was zunächst an seinen Reisezielen auffällt, ist, daß sie alle nur über das Wasser zu erreichen sind: Venedig, Sizilien, die Sireneninseln, Malta. Durchweg handelt es sich um Inseln; und das letzte Ziel, Nordafrika, ist jenseits des Meeres angesiedelt. $\mathrm{Da}$ hier in der Reihe der Orte ein imaginärer, mythischer, die Sireneninseln, erscheinen kann, überrascht nicht, denn es zeichnet den Absatz insgesamt aus, daß Schachs Fantasie sich mehr und mehr ins Irreale verliert. Geheimnisträchtigkeit und unwirklicher Charakter prägen die südliche Urlaubsszenerie; gerade in ihnen scheint ihre Anziehungskraft zu bestehen, und schließlich erstrebt Schach gar nicht mehr die Realität selbst, sondern nur noch ihre "Luftbilder und Spiegelungen «, die ihm die stumme Sirene mit ihrer laterna magica zeigt, wobei sich die Szenen selbst zunächst durch ihren ständigen Wechsel dem Zugriff zu entziehen scheinen.

Die Gestalten, denen Schach begegnen möchte, kommen in Märchen und Mythos vor, nicht in der Realität: Sirenen und Feen; und was sich Schach gegen Ende zu einer genauen Szene in einer Wüstenoase verdichtet, scheint aus den Märchen aus tausendundeiner Nacht entnommen. Wo Italien endet, beginnt ein Reich der Imagination, das voll von Todesassoziationen ist und keineswegs auf eine glückliche Ehe vorausdeutet. Das gilt schon von der Erwähnung der Sirenen. Ein Blick in ein gängiges Konversationslexikon der Zeit Fontanes vermittelt den populären Wissensstand von diesen mythologischen Gestalten:

Sirenen (Seirenes), Töchter des Phorkys oder des Acheloos und einer Muse, bei Homer zwei, in späterer Sage drei Jungfrauen, die auf einem Eiland zwischen der Insel der Kirke und der Skylla, auf einer Strandwiese, umgeben von bleichenden Gebeinen, durch ihren Gesang Vorübersegelnde anlocken, um sie zu verderben. Als Odysseus vorbeifuhr, verklebte er die Ohren seiner Gefährten mit Wachs und ließ sich selbst an den Mast binden, um sie ohne Gefahr zu hören. Die Argonauten schützte Orpheus durch seinen Gesang vor dem Zauber. Da sie nur so lange leben sollten, bis einer durch ihren Gesang unbetört bliebe, stürzten sie sich wegen Odysseus oder Orpheus ins Meer und wurden in Klippen verwandelt. Spätere Zeit lokalisierte sie auf den Sirenusen bei Neapel oder auf Capri oder dem sizilischen Vorgebirge Pelorum. Im Volksglauben der Griechen sind die s. dämonische Mischwesen mit Vogelleibern und Menschenköpfen, die, ursprünglich die abgeschiedene Seele selbst bedeutend, als Totenvögel erscheinen und auf Grabdenkmälern, oft klagend 
oder singend oder mit Instrumenten, häufig dargestellt sind, wobei die Vorstellung von den musizierenden s. auf den Einfluß der homerischen Sage zurückzuführen ist. ${ }^{4}$

Die enge Beziehung der Sirenen zum Totenreich - ob sie nun die toten Seelen darstellen oder begleiten oder ob sie die Menschen in den Tod locken ist das vorherrschende Element dieses Mythos, der zudem eine Gedankenverbindung auslöst, die wenig später mit dem noch zu diskutierenden Stichwort »Hyperboreer« verstärkt wird: Die Sirenen haben Selbstmord begangen, indem sie sich ins Meer stürzten - ein Schicksal, das symbolisch Schach selbst in seiner Reisefantasie erleidet. Aber darüber hinaus spiegelt seine Wendung von der singenden zur stummen Sirene seine Distanzierung von Victoire und der Ehe, denn er möchte zunächst die Sirene singen hören, ohne von ihr in den Untergang gelockt zu werden, und hofft bei diesem bisher nur Odysseus geglückten Unternehmen auf Victoires Vertrauen und Unterstützung. Ist sie selbst die singende Sirene, mit der er im zweiten Kapitel gemeinsam musiziert hat, und zwar ein Lied, das von dem Gegensatz von Kälte und Wärme und dem Ungenügen am Irdischen handelt? Es fällt auf, daß die einzige Nennung von Victoires Namen in der Reisefantasie in Anführungsstriche gesetzt ist und damit einen gewissen distanzierten Zitatcharakter bekommt. Daß Schach sich darin gerade Victoires Urteil anheimgibt, wird vollends zur ironischen Geste, wenn gleich darauf die stumme Sirene - ist sie der Tod? - der singenden gegenüber die Oberhand über Schachs Einbildungskraft gewinnt, weil sie »fast noch verführerischer locke«. In dem afrikanischen Paradies jedenfalls, das sie Schach vorgaukelt, kann Victoire keine Rolle mehr spielen. Die Gegenwelt zur Ehe hat im Laufe der Reisevision in seiner Vorstellung gesiegt; oder anders gesagt: Schach ist, da es sich um den Vorabend seiner Eheschließung handelt, verloren.

Welches nämlich ist seine Vision von Nordafrika, dem Ziel seiner Reisewünsche? Eine Fantasie, die Victoire warnen müßte, denn ebenso wie er in der Tempelhofer Kirche am Grab des Tempelritters, der bezeichnenderweise wohl gar keiner ist, seine zölibatären und militärischen Ideale und damit die ihm gemäße Lebensform verraten hat, tut er es auch hier: Er sieht in einer Oase die »Schar der Männer« im Kreise sitzen, die sich eben noch als »ermüdeter Zug über den gelben Sand« der Wüste geschleppt hat. Nun, wo die Mühsal überstanden ist, geben sie sich einmal einer in Schachs Zeit exklusiv männlichen Beschäftigung hin, dem Rauchen, ${ }^{5}$ und sie werden zum anderen von »schwarz und braunen Mädchen, ihre Flechten gelöst und wie zum Tanze geschürzt « umtanzt, eine zu Fontanes Zeit wohl gewagte und Schach gerade darum enthüllende Vision: Die Männer wie bei Louis Ferdinands Diner, wo man über Frauen diskutiert, gelassen sitzend und von orgiastisch animierenden Mädchen umtanzt, die auf durchaus doppeldeutige Weise »die Becken« 
heben; die Männer von den Frauen umworben und nicht umgekehrt; die Frau als Zeitvertreib, die in den geschlossenen und ernsthaften Kreis der Männer zwar nicht eindringen kann, sich aber als sexuelles Spielzeug aufreizend anbietet. Kein Wunder, daß sich dies - geheimnisvoll, verführerisch und unwirklich zugleich - nur jenseits des Meeres und als Fata Morgana abspielen kann, denn Schach ist es in der Realität ja gerade mißglückt, sein Verhältnis mit Victoire auf dieser Stufe zu halten. Das lebendig ausgemalte Bild, das Victoire zunächst aufjubeln läßt und sie dann »bang und düster« berührt, hat tragische Untertöne des Scheiterns: Es ist die Vision der Freiheit, wie Schach sie versteht; der Freiheit, die er zu verlieren im Begriff ist. Kein Wunder auch, daß dieses Bild erst lacht, dann schweigt, dann schwindet. Da es den erotischen Wunschtraum eines Junggesellen darstellt, der sich in der muselmanischen Welt wähnt, wo der Mann mehrere Frauen haben darf, nicht um die christlich-monogame Vision eines Bräutigams an der Schwelle zur Ehe, widerruft Schach mit der Fantasie unbewußt schon seine Entscheidung, Victoire zu heiraten; und so verwundert es denn auch nicht, da dies das Ziel seiner Träume ist, daß Schach seine Frau zunächst in die Fantasie seiner Hochzeitsreise gar nicht einschließt und sich erst bewußt dazu zu zwingen scheint, auch in ihrem Namen zu sprechen: "Er wollte nach Sizilien hinüber [...] Und dann wollten sie nach Malta.«

Viel eher als eine Hochzeitsreise ist dies die Flucht des Offiziers in den kolonialen Dienst, weg von den Liebes- oder Schuldenverpflichtungen, wie es zur Zeit des alten Fontane gang und gäbe war, der selbst Gestalten in seinen Romanen mit diesem Ausweg aus ihren Problemen spielen läßt. Roswithas Brief an Innstetten in Effi Briest bewegt diesen dazu, Wüllersdorf zu gestehen, er ginge am liebsten weg von hier, weg und hin unter lauter pechschwarze Kerle, die von Kultur und Ehre nichts wissen«. Aber Wüllersdorf antwortet nüchtern: »Quer durch Afrika, was soll das heißen? Das ist für 'nen Leutnant, der Schulden hat.« (EB 288) Der Leutnant mit Schulden ist Leo Poggenpuhl in Die Poggenpuhls. Was er sagt, klingt beinahe, als wäre es Teil von Schachs Nordafrikavision: »wer aber nichts hat, wer immer in einer Wüste Sahara lebt, der kann ohne Fata Morgana mit Palmen und Odalisken und all dergleichen gar nicht existieren. (DP 30) Zum Entsetzen der alten Dienerin Friederike malt er dann aus: »Wenn es aber nichts wird, na, dann Friederike, dann müssen die Schwarzen ran, das heißt die richtigen Schwarzen, die wirklichen, dann muß ich nach Afrika.« (DP 33) Im Falle Schachs würde es sich bei der Flucht nach Afrika allerdings um einen Anachronismus handeln, denn 1806 existierte die Möglichkeit, in den Kolonialdienst zu entfliehen, natürlich noch nicht.

Sucht man in Fontanes Werk nach einer Schachs Reisefantasie verwandten Szene, dann drängt sich, so eigenartig das auf den ersten Blick erscheinen mag, der Vergleich mit dem Selbstmord des Grafen Waldemar von Haldern in 
Stine auf, denn er und Schach sind die einzigen jungen Männer in Fontanes Romanen, die sich das Leben nehmen. Was erlebt Graf Haldern, bevor er Gift nimmt, sich in seinen Stuhl zurücklehnt und die Augen schließt ...? Von Stine abgewiesen, die ihn weder heiraten noch mit ihm ein neues Leben in Amerika beginnen will, geht er zu Fuß zu seiner Wohnung zurück (15. Kapitel) und nimmt dabei die Wirklichkeit rund um sich herum mit einer Intensität wahr wie nie zuvor: "Nichts entging ihm." Schon ein Blick auf die Verben der Szene macht die angespannte Aufmerksamkeit Waldemars deutlich: "Er zählte die Schläge (der Turmuhr) [...] musterte [...] betrachtete [...] sah [...] musterte $[\ldots]$ bemerkte $[\ldots]$ sah $[\ldots]$ sah $[\ldots]$. « Was sich im Text auf einen einzelnen Sinneseindruck bezieht, charakterisiert sein Verhalten insgesamt: »Unser Kranker sog das alles in vollen Zügen ein.« (S $85 \mathrm{ff}$.)

Umgekehrt wie Schach also flieht er nicht ins Fantastische, in die Fata Morgana, sondern in das, woran ihm durch seine Krankheit die Teilnahme versagt ist. Das ländliche Leben, vor dem Schach erschrickt, ist sein Wunschtraum: "Ich sehne mich danach, einen Baum zu pflanzen oder ein Volk Hühner aufsteigen oder auch bloß einen Bienenstock ausschwärmen zu sehen.« Das wirkliche, alltägliche Leben zieht ihn, als er vollends davon Abschied zu nehmen im Begriff ist, mit besonderer Stärke an. Und damit zeigt sich: Obwohl Waldemars Eindrücke sachlich denen Schachs entgegengesetzt sind, ist die Methode bei beiden Szenen die gleiche: Beide wenden sich dem ihnen sonst Fremden zu (»Schach [...] hing mit einer ihm sonst völlig fremden Phantastik [...]«). Das, was ihre bisherige Existenz nicht enthielt, tritt nun in den Vordergrund. Schach, sonst als Mann von Welt in der Realität zu Hause und ein gesuchter Gesellschafter, bricht aus ihr aus, und Waldemar, sonst von der Realität aufgrund seiner Krankheit ausgeschlossen, wendet sich ihr nun zu. Aber die Verbindungen zwischen den beiden Episoden sind noch enger: Zunächst befestigt sich der Gedanke an den Tod in ihnen, als ihr Bewußtseinszustand herabgemindert ist. Wie Schach im Wuthenow-Kapitel im leise schaukelnden Kahn in Schlaf fällt (»und er schlief ein«, 98), so setzt sich Haldern während seines Spazierganges ermüdet »auf eine Bank « und »fiel in Traum und Vergessen « (S 86). Wenn um Waldemar dabei eine Atmosphäre von Resignation und Melancholie herrscht, Schach aber bis unmittelbar vor seinem Tod Kraft und Selbstvertrauen ausstrahlt, dann erklärt in beiden Fällen der Text selbst, warum. Als Schach in seinem Boot aufwacht, ist der Tag »inzwischen angebrochen. Über dem First des Wuthenower Herrenhauses hing die Sonne« (98).

Bei Waldemar umgekehrt wird es Abend. Zu Hause angekommen, blickt er aus dem Fenster: »Die Sonne war im Niedergehen [...] Und er sah in die röter werdende Glut, bis endlich der Ball gesunken und volle Dämmerung um ihn her war.« Während er dieses Bild betrachtet, erinnert er sich an seinen ersten Besuch bei Stine, wo er ebenfalls den Sonnenuntergang beobachtet hat, und 
") Wie damals<, sprach er vor sich hin « (S 86 f.), eine Bemerkung, die den psychologischen Gegensatz der beiden Selbstmörder weiter verdeutlicht: Wie Waldemar zurückblickt, so Schach im Angesicht des Todes nach vorn: »Reisepläne und Reisebilder « haben es ihm angetan. Es stimmt mit diesen Charakterunterschieden der >Helden< überein, daß Schach sich seinem Mädchen entzieht, während umgekehrt Haldern der Abgewiesene ist; wie auch Schach sich erschießt, während Haldern zum unheroischen Gift greift. Hat es mit diesem Gegensatz zu tun, daß Waldemar im Süden war, für Schach aber der Süden das Ziel des "in Nebel und Schnee geborenen Hyperboreer[s] « ist? Dieses Wort läßt aufmerken, denn Schach bezeichnet sich damit schon zum zweiten Mal. Schon in dem Billett, mit dem er im 4. Kapitel die Damen Carayon zur Spazierfahrt einlädt, schreibt er: "Alles in allem ein so schöner Tag, wie sie der April uns Hyperboreern nur selten gewährt.« (27)

Wieder kann der entsprechende Artikel aus dem schon zitierten Konversationslexikon den assoziativen Hintergrund für dieses Stichwort herstellen, das Schach selbst zweimal auf sich bezieht und das, von Fontane ohne Zweifel in künstlerischer Absicht verwendet, ${ }^{6}$ der indirekten Charakterisierung des Helden dient, auf den es außerordentlich aufschlußreiche Schlaglichter wirft:

Hyperboreer, ein fabelhaftes Volk, das durch die Überlieferung mit den Tempeln in Delos, Delphi und Tempe in Verbindung gebracht worden ist. Der Name bedeutet, daß es hoch im Norden, noch >über den boreas hinaus<, wohnt und daher von dem kalten Nordwind nicht getroffen wird. Während Herodot und Strabon die Existenz eines solchen Volkes bezweifeln, suchen die meisten spätern Dichter und Geographen den Hyperboreern im Norden der Erde bestimmte Wohnsitze anzuweisen. Allenthalben erscheinen die $h$. in ihrem milden, sonnigen und fruchtbaren Land als ein gliickseliges Volk, ausgezeichnet durch Reinheit der Sitten und Frömmigkeit und von langer Lebensdauer. Sie wohnten in Hainen, lebten von Baumfrüchten und kannten weder Krieg noch Streit. Mit größtem Eifer lagen sie dem Kultus des Apollon ob, der vom Beginn des Frühlings bis in den Sommer hinein bei ihnen zu verweilen pflegte, und wurden weder durch Krankheit noch durch kraftloses Alter gestört. Dem heiligen Volk war ein tausendjähriges Alter beschieden, und erst, wenn sie des Lebens satt waren, starben sie freiwillig durch einen Sprung in das Meer. ${ }^{7}$

Vergleicht man Schachs Vorstellung des »in Nebel und Schnee gebornen Hyperboreer[s]« mit dieser lexikalischen Erläuterung, dann ist offensichtlich, daß Fontane die klimatischen Bedingungen des fabelhaften Volkes geändert hat: Eiszeit in Preußen statt ewiger Sonne. Aus dem naiven echten Hyperboreer ist im Sinne des Romanthemas ein sentimentalischer Schach geworden. 
Denn der Mythos vom hyperboreischen Volk ist ein Mythos vom goldenen Zeitalter, vom einfachen, moralisch integren Leben, das Schach und das PreuBen seiner Zeit, das er repräsentiert, gerade verloren haben. Das selbstgenügsam-unkorrumpierte, »Reinheit der Sitten und Frömmigkeit«, sieht Schach wohl in der Ehe mit Victoire als Aufgabe vor sich, ja, es macht die Substanz seiner Fantasie eines ländlichen, ihn bestürzenden Ehelebens im 13. Kapitel aus. Aber er ist nicht bereit, seine Pflicht zu erfüllen, weil er weiß, daß ihn die Sehnsucht verzehren wird, diesem Dasein zu entgehen: "Kenn ich nicht die Gebote? Was mir fehlt, ist bloß die Lust, ihnen zu gehorchen.« (102)

So bekommt der Mythos utopischen Charakter: Er signalisiert, was Schach und das Preußen von 1806 sein sollten, aber nicht sind. Es versteht sich damit von selbst, daß Schachs Hyperboreertum teilweise ein Wunschtraum ist, dem gegenüber er versagt. Der kurze Dialog zwischen Nostitz und Bülow bei "Sala Tarone « im 3. Kapitel reflektiert dieses Doppelgesicht des Helden genau: »Er ist immerhin einer unserer Besten.«»Um so schlimmer.« (24)

Daß Victoires Häßlichkeit bei seinen Ehebildern in Wuthenow eine solche Rolle spielt, hängt durchaus mit seinem Hyperboreertum zusammen, denn wie diese huldigt er als Ästhet »mit großem Eifer« Apollo, dem Gott der Schönheit, und es ist bezeichnend, daß er Victoire erst verführt, als er sie in Gedanken mit dem Typ der von Prinz Louis Ferdinand gepriesenen »beauté du diable « identifiziert hat. Aber die Implikationen des Begriffs Hyperboreer enthalten darüber hinaus auch den Schlüssel zur Reisefantasie Schachs, in der das Worte wiederverwendet wird. Da die Hyperboreer wie die schon erwähnten Sirenen ihrem Leben durch einen Sprung in das Meer ein Ende machen, bekommt der Leser indirekt schon im 4. Kapitel einen Hinweis auf Schachs Tod, und alle die von ihm ersehnten Orte von Venedig bis zur Oase in Nordafrika wird er, eben weil er sich wie ein Hyperboreer verhalten wird, nie erreichen. Zwischen ihnen und ihm liegt in jedem Falle das Meer, in das er sich - symbolisch - stürzen wird.

Ähnlich erträumt in Unwiederbringlich Graf Holk sich im weihnachtlichkalten Flensburg eine Hochzeitsreise nach Italien, die ebenfalls nicht stattfindet. Als er nach Italien kommt, ist er heimatlos, da seine Frau von ihm geschieden ist und Ebba Rosenberg seinen Heiratsantrag abgelehnt hat.

Im Gegensatz zu Schach ist Waldemar von Haldern im Süden gewesen, spricht aber interessanterweise nur ein einziges Mal davon, um zu bemerken: »In Sorrent, mit dem Blick auf Capri, war ich elend und unglücklich.« (S 79) Nur von seinem Onkel wird er wiederholt darauf angesprochen. Schon bei der Witwe Pittelkow sagt er von seinem Neffen: „Er reist seit fünf Jahren in Italien umher, $[\ldots]$ « (S 25). Später nennt er ihn einen »Italianissimus«, obwohl er gleich darauf spürt: »Diese Dinge stören dich.« (S 64) Erst beim letzten Gespräch zwischen Onkel und Neffen gibt der alte Graf dem Leser einen Hinweis, warum er an Italien hängt, auch Waldemars Gedanken wieder dorthin 
lenken und nichts von Amerika, dem neuen Ziel Waldemars, wissen möchte: »Und den Tag danach schreibst du ihr einen Abschiedsbrief und trittst deine dritte Römerfahrt an. Rom paßt ohnehin für die Halderns, alt zu alt.« (S 69)

Italien, für Waldemar das verleugnete Ziel, ist für Schach das ersehnte. Die Irrealität seiner noch darüber hinaus, immer weiter nach Süden gehenden Wünsche drückt sich schon darin aus, daß seine ganze Vision in indirekter Rede wiedergegeben ist, was ihr allerdings nichts von ihrer Farbigkeit und Exotik nimmt. Zumal gegen Ende besteht der Text fast nur noch aus Konjunktiven: »Und mitunter sei's, als lach' es. Und dann schwieg' und schwänd' es wieder.« Die Sätze, in denen die Apokope des >-e< selbst das Schwinden anzuzeigen scheint, sprechen nur noch von einem undefinierten Ziel »es«. So entgleitet Schach schließlich die vorher evozierte präzise Szene in der Oase ins Unpersönliche, Ungreifbare, Mysteriöse, ins ganz und gar Unwirkliche. Der dann folgende Satz bestätigt den Eindruck, daß Schachs fantastische Reisewünsche eigentlich sein Wegsehnen aus der Realität, aus dem Leben verraten, denn offen wird darin das Ziel als bloße »Spiegelung aus der geheimnisvollen Ferne « bezeichnet. Die »Lebhaftigkeit seiner Schilderung " hat die fernen Ziele nicht näher gebracht, sondern ihr hoffnungsloses Fernsein bestätigt. Schach hat Wuthenow endgültig verlassen; er ist auf dem Weg nach Venedig.

So indirekt, wie diese Formulierung auf den eigentlichen Vorgang nur anspielt, ist Fontanes Verfahren insgesamt in dem diskutierten Abschnitt. Da schon ein zeitgenössischer Kritiker in seiner Besprechung von Schach von Wuthenow Fontane als "Gesellschaftslandschafter ${ }^{8}$ bezeichnet hat, mag es angebracht sein, ihn im Hinblick auf Schachs Reisefantasie als >Seelenlandschafter zu charakterisieren: Psychographie nicht im Sinne der Seelenmalerei, sondern im Sinne der Darstellung dessen, was das seelische Geschehen mit auszudrücken vermag. Gerade dadurch erreicht der Text seinen Beziehungsreichtum, seine Dichte und seine Diskretion, die sich mit bloßen Andeutungen und Anspielungen begnügt. Fontanes Gestaltung der Reisewünsche Schachs ist ein vollendetes Beispiel konzentriertester Symbolik innerhalb seines durchaus realistischen Erzählens.

Anmerkungen

${ }^{1}$ Benno von Wiese neigt in seiner Interpretation (in: Die Deutsche Novelle von Goethe bis Kafka. 2. Bd. Düsseldorf 1965, S. 236-260) eher zum 
Nacherzählen als zur Analyse (s. 258 f.). Demetz beschränkt sich auf einen kurzen Hinweis (S. 141). Cordula Kahrmann (Idyll im Roman: Theodor Fontane. München 1973) sieht im Sinne der Thematik ihrer Arbeit Schachs Vision als ein Idyll, geht aber kaum ins Detail (S. 115 f.). Siehe dazu Anm. 5.

${ }^{2}$ Hans R. Vaget, »Schach in Wuthenow: >Psychographie< und >Spiegelung im 14. Kapitel von Fontanes >Schach von Wuthenow «", in: Monatshefte LXI (1969) No. 1. S. 1-14.

${ }^{3}$ Demetz, S. 136.

${ }^{4}$ Meyers Grosses Konversationslexikon. Leipzig und Wien ${ }^{6} 1902,18$. Bd., S. $499 \mathrm{f}$.

${ }^{5}$ Kahrmann (a. a. O.) schreibt zu dieser Stelle: »Einzelzüge aus der Schilderung des Besuchs beim Prinzen (die rauchenden Männer) und des Massowschen Kinderballs (die Gesten der tanzenden Mädchen) tauchen hier wieder auf [...].« Der Hinweis auf die rauchende Gesellschaft beim Prinzen mag als Parallele angebracht sein. Den Kinderball und damit Victoire mit den Tanzenden zu verbinden, scheint mir an Funktion und Sinn dieser Szene völlig vorbeizugehen.

${ }^{6}$ Fontane verwendet das Worte in seinen Romanen sonst wohl nur im 29. Kapitel von Quitt in der Formulierung »diesen preußisch-hyperboreischen Tannenbaumkultus.« (Q 205)

${ }^{7}$ Meyers Grosses Konversationslexikon. Leipzig und Wien ${ }^{6} 1905,9$. Bd., S. 707.

${ }^{8}$ Eduard Engel, Rezension von Schach von Wuthenow, in: Das Magazin für die Literatur des In- und Auslandes. 1882, Nr. 52, S. 720-722. Zitiert nach: P.-P. Sagave: Theodor Fontane: Schach von Wuthenow. Ullstein Taschenbuch Bd. 5023, S. 183 (= Dichtung und Wirklichkeit). 